\title{
Optimization of MANET Connectivity Via Smart Deployment/Movement of Unmanned Air Vehicles
}

\author{
Zhu Han, Member, IEEE, A. Lee Swindlehurst, Fellow, IEEE, and K. J. Ray Liu, Fellow, IEEE
}

\begin{abstract}
Unmanned air vehicles (UAVs) can provide important communication advantages to ground-based wireless ad hoc networks. In this paper, the location and movement of UAVs are optimized to improve the connectivity of a wireless network. Four types of network connectivity are quantified: global message connectivity, worst-case connectivity, network bisection connectivity, and $k$-connectivity. The problems of UAV deployment and movement are formulated to improve the different types of connectivity. Both problems are $N P$-hard. For the deployment case, some heuristic adaptive schemes are proposed to yield simple but effective solutions. In addition, a closed-form solution for the two-node one-UAV case is provided. For $k$-connectivity, we propose an algorithm that improves connectivity using Delaunay triangulation. To optimize the UAV movement, an algorithm that tracks changes in the network topology is constructed. The simulation results show that by only deploying a single $\mathrm{UAV}$, the global message network connectivity and the worst-case network connectivity can be improved by up to $109 \%$ and $60 \%$, respectively. The network bisection connectivity and the $k$-connectivity can also be significantly improved.
\end{abstract}

Index Terms-Ad hoc network, network connectivity, unmanned air vehicle (UAV).

\section{INTRODUCTION}

$\mathbf{U}$ NMANNED air vehicles (UAVs) are playing increasingly prominent roles in the nation's defense programs and strategy. While drones have been employed in military applications for many years, technological advances in microcontrollers, sensors, and batteries have dramatically increased their utility and versatility. Traditionally, emphasis has been placed on relatively large platforms such as Global Hawk and Predator, but increasing attention has recently been focused on small "mini-UAVs" (MUAVs) that offer advantages in flexibility and cost [1]-[3]. An example of an experimental MUAV built and tested at Brigham Young University is depicted in Fig. 1. Because of their small size, they are difficult for others to detect and track, and they are able to more easily avoid threats in the environment they fly through. As a result, they can fly at much lower altitudes, on the order of tens or hundreds of feet, and collect much more precise "localized" data. They are

Manuscript received September 15, 2008; revised December 18, 2008. First published February 24, 2009; current version published August 14, 2009. The review of this paper was coordinated by Prof. B. L. Mark.

Z. Han is with the Department of Electrical and Computer Engineering, University of Houston, Houston, TX 77004 USA.

A. L. Swindlehurst is with the Department of Electrical and Computer Science, University of California at Irvine, Irvine, CA 92697 USA.

K. J. R. Liu is with the Department of Electrical and Computer Engineering, University of Maryland, College Park, MD 20742 USA.

Color versions of one or more of the figures in this paper are available online at http://ieeexplore.ieee.org.

Digital Object Identifier 10.1109/TVT.2009.2015953

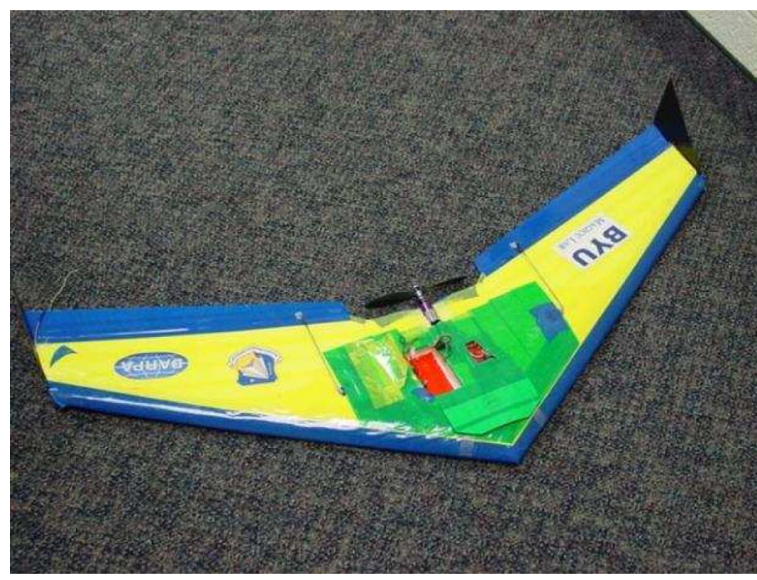

Fig. 1. Miniature UAV built and flown at Brigham Young University.

significantly cheaper and easier to fly, easily carried, and can often be launched by an individual in any kind of terrain without a runway or special launching device.

Due to their mobility and elevation, UAVs equipped with communication capabilities can provide important advantages to ground-based ad hoc networks. Their use in routing, medium access control, and scheduling applications has been detailed in [4]-[7]. These studies have primarily been heuristic and have focused on simulations to qualitatively assess the benefits of UAV-assisted networks. In this paper, we take a mathematical approach to positioning and flying a UAV over a wireless ad hoc network to optimize the network's connectivity for better quality of service (QoS) and coverage. We assume a single UAV flying over a connected network with estimates of the positions and velocities of the network nodes. The UAV itself acts as a node in the network and can generate, receive, or forward data packets to other nodes to improve network connectivity.

Network connectivity has recently been widely studied, particularly in the context of mobile ad hoc networks (MANETs). In [8], it is shown that the introduction of a sparse network of base stations can significantly help in increasing the network connectivity. In [9], the authors determine the critical power that a node in the network needs to transmit to ensure that the network is connected with probability 1 , as the number of nodes in the network goes to infinity. Miller [10] calculates the probability that two nodes are connected by a two-hop path rather than a direct connection. In [11], the authors study how large the transmitting range must be to ensure that the network is connected with high probability. In [12], it is shown that in a network with $K$ randomly placed nodes, if each 
node is connected to less than $0.074 \log K$ neighbors, then the network is asymptotically disconnected with probability 1 as $K$ increases; if each node is connected to more than $5.1774 \log K$ neighbors, then the network is asymptotically connected with probability approaching 1 as $K$ increases. In [13], the authors study how to deploy as few additional nodes as possible so that the augmented network is connected. Khuller [14] studies the Connectivity Augmentation problem and determines a set of edges of minimum weight to be inserted so that the resulting graph is $k$-connected. In [15] and [16], analytical expressions that enable the determination of the required range that creates, for a given node density, an almost surely $k$-connected network are derived.

In this paper, we quantify four types of network connectivity for the UAV-assisted MANET problem. First, global message connectivity is defined as the highest possible probability of successfully propagating one message to all nodes in the network. This connectivity measure represents how well a command can be delivered to all nodes. Second, worst-case connectivity is defined to partially measure how close a network is to being divided. Third, we define network bisection connectivity, which quantifies the cost of dividing the network. Finally, $k$-connectivity is defined to quantify how many nodes must fail before the network becomes disconnected. The first two types of connectivity are based on the theory of spanning trees, the third type is based on spectrum graph theory, and the fourth type is based on the max-flow min-cut theorem and Menger's theorem [17], [18]. Using these four definitions, we formulate the UAV deployment and movement problems, both of which are shown to be $N P$-hard. To solve the deployment problem, we first analytically study a two-node one-UAV case. Then, we develop a simple and heuristic algorithm with two types of initialization for optimally governing the UAV's position. Moreover, for $k$-connectivity, we propose an algorithm for improving connectivity using Delaunay triangulation (DT). Finally, we discuss two algorithms for determining how to adjust the velocity and direction of the UAV to optimize connectivity as the network topology changes. Simulation results demonstrate that the addition of one UAV can improve the global message connectivity and the worst-case connectivity by up to $109 \%$ and $60 \%$, respectively. We also study the improvement in network bisection connectivity and show that $k$-connectivity can be improved by almost 1 . Some preliminary results were presented earlier in [19].

This paper is organized as follows. In Section II, we describe the model for the system we consider, and in Section III, we present mathematical formulations of the connectivity definitions used as our performance metrics. Section IV addresses the UAV deployment problem by first finding an optimal solution to the two-node one-UAV case and then deriving simpler but effective heuristic solutions. Finally, we formulate the UAV movement problem. Simulation results are given in Section V, followed by conclusions in Section VI.

\section{UAV-Assisted Network Model}

We assume a single UAV flying over a wireless MANET that is able to securely obtain or estimate the locations and velocities of the randomly distributed mobile nodes in the network. In particular, we assume the UAV to possess the following information:

1) locations of all nodes $\left(x_{i}, y_{i}\right)$ from which the distance between any two nodes is calculated to be $D_{i j}=$ $\sqrt{\left|x_{i}-x_{j}\right|^{2}+\left|y_{i}-y_{j}\right|^{2}}$

2) estimates of the mobile nodes' speed and direction based on the knowledge of their locations at different times

$$
S_{i}=\frac{d x_{i}}{d t}+z \frac{d y_{i}}{d t}
$$

where $z=\sqrt{-1}$. Here, the real and imaginary values represent the $x$ and $y$ coordinates, respectively.

Suppose that there are $K$ mobile nodes plus one UAV denoted as node 0 in the MANET. The wireless channel response between any two nodes is denoted by $G_{i j}, i \neq j$. Suppose that node $i$ transmits with power $P_{i}$ and observes noise of average power $\sigma_{i}^{2}$ when operating as a receiver. In military applications, the bandwidth is sufficient. As a result, different users and UAVs use different frequencies. Therefore, we assume that interference is trivial. The received signal-to-noise ratio (SNR) $\Gamma_{i j}$ for the signal transmitted by the $i$ th node and received by the $j$ th node is thus

$$
\Gamma_{i j}=\frac{P_{i} G_{i j}}{\sigma_{j}^{2}} .
$$

Using the Rayleigh model, the channel gain can be expressed as

$$
G_{i j}=\frac{C_{i j}\left|h_{i j}\right|^{2}}{\left(D_{i j}\right)^{\alpha}}
$$

where $C_{i j}$ is a constant that takes into account the antenna gains and any propagation obstructions (shadowing), $h_{i j}$ accounts for multipath fading, $D_{i j}$ is the distance between node $i$ and node $j$, and $\alpha$ is the propagation loss factor.

Maintaining a minimal level of link quality requires an acceptably small packet loss, which in turn requires a sufficiently high received SNR. Suppose that the SNR threshold needed to obtain minimal link quality is $\gamma$. If we assume that the channel has fast Rayleigh fading $\left(h_{i j}\right.$ is complex Gaussian with zero mean and unit variance), then using (2) and (3), we calculate the probability of a successful transmission between node $i$ and node $j$ as

$$
P_{r}^{i j}\left(\Gamma_{i j} \geq \gamma\right)=\exp \left(-\frac{\sigma_{j}^{2} \gamma\left(D_{i j}\right)^{\alpha}}{C_{i j} P_{i}}\right) .
$$

Note that in the preceding expression, we have integrated out the fast Rayleigh fading, and therefore, the probability of a successful transmission should be interpreted in an average sense over multiple fading events. Because the transmission power is bounded, we will say that two nodes are connected if the probability defined in (4) is greater than or equal to some threshold $\delta$. Based on this definition, we construct a graph $G(K, \mathbf{A})$ to describe the connectivity of the network, where the matrix $\mathbf{A}$ is constructed as

$$
[\mathbf{A}]_{i j}= \begin{cases}1, & \text { if } P_{r}^{i j} \geq \delta \\ 0, & \text { otherwise. }\end{cases}
$$


We assume that the links are symmetric between all nodes, or, in other words, $P_{r}^{i j}=P_{r}^{j i}$, so that the resulting graph is undirected.

If every node in the network can reach any other node by either direct connection or routing via other nodes, then we say that the network is connected in the sense of (5). In this paper, we assume that the network is connected to begin with and concentrate on how to improve connectivity as the network evolves. This goal requires that there be some way of quantifying how well connected a given network is beyond the simple definition given in (5). The development of such connectivity measures is the goal of the following section.

\section{Network Connectivity Definitions}

In this section, we present four definitions of numerical network connectivity for the UAV-assisted network model: global message connectivity, worst-case connectivity, network bisection connectivity, and $k$-connectivity. The first two types of connectivity are based on the theory of spanning trees, the third type is based on spectrum graph theory, and the fourth type is based on the max-flow min-cut theorem and Menger's theorem [17], [18]. After defining these connectivity measures, in the next section, we show how they may be optimized through intelligent positioning of a UAV relay.

\section{A. Global Message and Worst-Case Connectivity}

In many applications, such as those involving military MANETs, it is important to keep all nodes in the network connected. For example, in battlefield scenarios, it is essential to propagate commands to all of the distributed soldiers and vehicles. A natural question in such cases is how to select which links are used to efficiently distribute information throughout the entire network such that all the nodes are connected and the overall network connectivity is maximized. The concept of a minimal spanning tree (MST) from graph theory provides an answer to this question.

Definition 1: A spanning tree for a given graph is a subgraph that is itself a tree and connects all the vertices of the graph together. A single graph can have many different spanning trees. A weight can be assigned to each graph edge that represents the "distance" between the two corresponding vertices, and the cost of a given spanning tree can be computed by summing all the weights of its edges. An MST or minimum weight spanning tree is then a spanning tree with cost that is less than or equal to the cost of every other spanning tree. The MST of a given graph may not be unique [17], [18].

For our application, to quantify each link's connectivity, we define the weight for each link as a function of the probability of a successful transmission, i.e.,

$$
W_{i j}=-\log P_{r}^{i j}
$$

where the minus sign is added to make the weight positive. Suppose node $i$ tries to communicate with node $j$ via node $l$. Because of the logarithmic form of the weights, the sum $W_{i l}+$ $W_{l j}$ will correspond to the weight associated with transmission

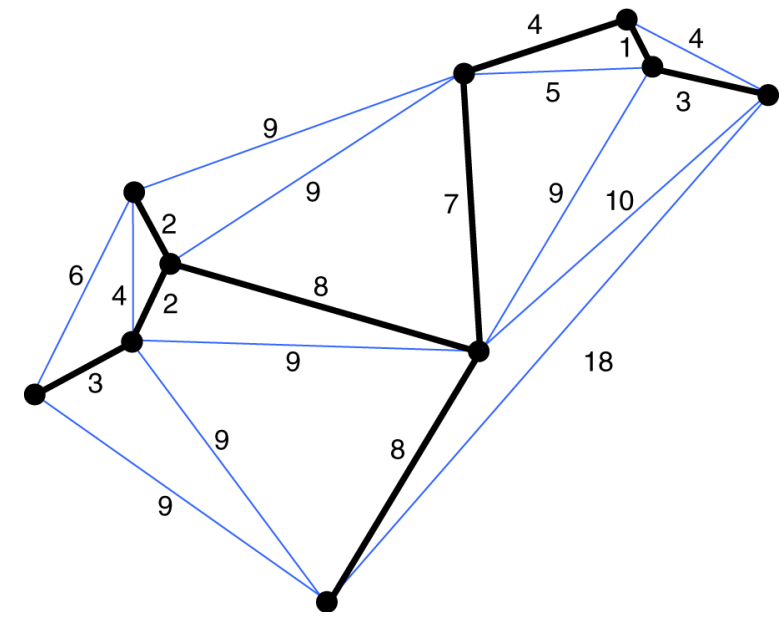

Fig. 2. Example of an MST, Steiner point, and Steiner tree.

between node $i$ and node $j$, which has a probability of success given by $P_{r}^{i l} P_{r}^{l j}$. The smaller the weight, the higher the probability of a successful transmission and, as a result, the greater the connectivity.

One example of a MANET and its MST is shown in Fig. 2. First note that, without considering the Steiner point, which we will discuss later, there are a total of ten nodes in the network. The possible connections between various pairs of nodes are marked with different weights, which correspond to the cost of transmission between them. Together, the bold links represent the MST that connects all of the nodes. To find the MST solution, a variety of polynomial-time algorithms are available, such as those proposed by Prim, Kruskal, or Chazelle [17], [18].

MSTs are widely used in wired networks to minimize transmission costs. Because of the broadcast nature of wireless communications, the transmissions of one node can be heard by many others. In [20], a pruning MST is proposed to yield energy-efficient broadcast and multicast trees. In our paper, however, we are interested in improving the connectivity and not in how to construct the spanning tree. The approaches discussed in the rest of this paper can be employed for any tree, like those in [20]. In what follows, we will let the matrix $\mathbf{A}^{\prime}$ represent the MST, where $\left[\mathbf{A}^{\prime}\right]_{i j}=1$ if the link from node $i$ to node $j$ is part of the MST, and $\left[\mathbf{A}^{\prime}\right]_{i j}=0$, otherwise.

We define the global message connectivity to be the probability that a message can successfully be transmitted to all nodes via the MST. Suppose that the MST has already been constructed with the weights defined in (6). Since the sum of the weights in the MST corresponds to the overall probability that a message is successfully transmitted via this MST, the global message (gm) connectivity may be computed as ${ }^{1}$

$$
U^{g m}=\sum_{i=1}^{K} \sum_{j=1}^{K}\left[\mathbf{A}^{\prime}\right]_{i j} W_{i j} .
$$

The smaller $U^{g m}$ is, the more likely it is that a message will be able to be delivered to all nodes in the network.

\footnotetext{
${ }^{1}$ Note that this claim is valid when the goal is to maximize the probability of a successful broadcast transmission and energy is not considered. If an energy constraint is necessary, then alternative techniques are required (cf., [20]).
} 
Next, we define the worst-case connectivity as the lowest probability of a successful transmission for any of the links on the MST. This type of connectivity represents how "close" a network is to being divided in two. Computing this connectivity measure is equivalent to simply finding the weight of the worstcase MST edge as

$$
U^{w c}=\max W_{i j}
$$

over $\left[\mathbf{A}^{\prime}\right]_{i j}=1 \forall i, j$. Again, it is desirable that $U^{w c}$ be as small as possible.

\section{B. Network Bisection Connectivity}

In this section, we define another type of network connectivity (which is referred to as bisection connectivity) that describes the likelihood that the network could be divided into two groups. As we subsequently show, network bisection connectivity is based on the eigenvalues of the graph's modified Laplacian matrix. In this case, we use a different definition for the weight of the link between node $i$ and node $j: w_{i j}=P_{r}^{i j}$. Note that in this definition, the weights do not correspond to "distance" as before; here, a large value (near unity) for $w_{i j}$ indicates two nodes that are strongly connected. Before discussing how to quantify this type of connectivity, we provide the following two definitions.

Definition 2: The modified Laplacian matrix $\mathbf{L}(G(K, \mathbf{A}))$ of the graph $G(K, \mathbf{A})$ is a positive semidefinite $K \times K$ symmetric matrix with one row and column for each node defined by

$$
[\mathbf{L}]_{i j}= \begin{cases}\sum_{k=1}^{K} w_{i k}, & \text { if } i=j \\ -w_{i j}, & \text { if } i \neq j \text { and } \exists \text { edge }(i, j) \\ 0, & \text { otherwise }\end{cases}
$$

for $i, j=1, \ldots, K$.

Definition 3: The Fiedler value and the Fiedler vector are the eigenvalue and the eigenvector, respectively, corresponding to the second smallest eigenvalue of the modified Laplacian matrix $\mathbf{L}$ of a graph $G(K, \mathbf{A})$. Note that the modified Laplacian matrix is positive semidefinite, the smallest eigenvalue of $\mathbf{L}$ is always zero, and the corresponding eigenvector is given by $1 / \sqrt{K}$, which is a vector whose entries are all equal to $1 / \sqrt{K}$.

The basic idea for bisection connectivity comes from the minimum cut tree (MCT) problem [23], which is widely used for clustering. In an MCT, the graph or network is divided/cut into two parts by minimizing a normalized sum of weights on some $c u t$, where cut is defined as a set of edges that are removed to divide the network into two disconnected subnetworks. One way of quantifying the cost of separating the network is referred to as expansion. Let a given graph $G$ be divided into two subnetworks $(\Xi, \bar{\Xi})$. The expansion of this cut is defined to be

$$
\psi(\Xi)=\frac{\sum_{i \in \Xi, j \in \bar{\Xi}} w_{i j}}{\min \{|\Xi|,|\bar{\Xi}|\}}
$$

where $|\Xi|$ and $|\bar{\Xi}|$ are the number of nodes in the separated graphs.

Based on the preceding discussion, we define the network bisection connectivity as the minimum expansion over all possible network cuts. Network bisection connectivity is more general than worst-case connectivity, since it identifies the set of nodes that are most weakly connected to the network, rather than just the weakest link of the spanning tree. Given two disconnected subnetworks $\Xi$ and $\bar{\Xi}$, let $q_{i} \in\{1,-1\}$ indicate whether the $i$ th node is grouped into $\Xi$ or $\Xi$, respectively. It is easy to show that $\min \{|\Xi|,|\bar{\Xi}|\}=(1 / 2)\left(K-\left|\sum_{i} q_{i}\right|\right)$. Assigning $w_{i j}=P_{r}^{i j}$, the numerator of (10) can be written as

$$
\sum_{i \in \Xi, j \in \bar{\Xi}} w_{i j}=\frac{1}{8} \sum_{i=1}^{K} \sum_{j=1}^{K}\left(q_{i}-q_{j}\right)^{2} P_{r}^{i j}
$$

where the factor of 8 comes from the fact that $\left(q_{i}-q_{j}\right)^{2}=4$ if $i \in \Xi, j \in \Xi$, and the fact that the double sum counts each link between $\Xi$ and $\bar{\Xi}$ twice. Putting these expressions together, the network bisection connectivity can be expressed as the following minimization over all possible network cuts:

$$
U^{n b}=-\min _{q_{i} \in\{-1,1\}} \frac{\sum_{i, j}\left(q_{i}-q_{j}\right)^{2} P_{r}^{i j}}{4\left(K-\left|\sum_{i} q_{i}\right|\right)} .
$$

The presence of the minus sign is to make the definition of $U^{n b}$ consistent with $U^{g m}$ and $U^{w c}$, where reducing the value of the connectivity measure is considered desirable. In this case, we want the "cost" of dividing the network in two (the expansion) to be as large as possible or the negative cost to be as small as possible.

The search in (11) over the set of integer indices $\left\{q_{i}\right\}$ is $N P$ hard [23]. To reduce the complexity, we relax the integer constraint on the entries of the vector $\mathbf{q}=\left[q_{1}, \ldots, q_{K}\right]$ and assume that $\mathbf{q}$ has real-valued entries subject to the norm constraint $\mathbf{q}^{T} \mathbf{q}=K$. We further define $\epsilon$ to be the difference between the sizes of two separated sets $\left(\epsilon=\sum_{i} q_{i}=|| \Xi|-| \bar{\Xi}||\right)$ and introduce the following relaxed version of (11) as our measure of network bisection connectivity:

$$
\begin{aligned}
\hat{U}^{n b} & =-\min _{\mathbf{q} \in \mathbb{R}^{\mathbb{K}}} \frac{\mathbf{q}^{T} \mathbf{L} \mathbf{q}}{2(K-\epsilon)} \\
\text { s.t. } \mathbf{q}^{T} \mathbf{q} & =K,\left|\mathbf{q}^{T} \mathbf{1}\right| \leq \epsilon .
\end{aligned}
$$

To obtain the solution to (12), we first prove the following theorem and its corollary.

Theorem 1: Let $\mathbf{B} \in \mathbb{R}^{\mathbb{K} \times \mathbb{K}}$ be a symmetric nonnegative definite matrix with eigenvalues $\Lambda_{1}<\Lambda_{2} \leq \cdots \leq \Lambda_{K}$ and corresponding eigenvectors $\mathbf{v}_{1}, \mathbf{v}_{2}, \ldots, \mathbf{v}_{K}$ with norm 1 . Then, for a fixed $0 \leq \beta<1$, the following problem:

$$
\begin{gathered}
\min \quad \mathbf{x}^{T} \mathbf{B} \mathbf{x} \\
\text { s.t. } \mathbf{x}^{T} \mathbf{x}=1, \quad\left|\mathbf{x}^{T} \mathbf{v}_{1}\right| \leq \beta
\end{gathered}
$$

has the solution

$$
\mathbf{x}=\beta \mathbf{v}_{1}+\sqrt{1-\beta^{2}} \mathbf{v}_{2}
$$

Proof: Write the eigen-decomposition of the matrix $\mathbf{B}$ as $\mathbf{B}=\mathbf{X} \Sigma \mathbf{X}^{T}$, where $\Sigma=\operatorname{diag}\left(\Lambda_{i}\right)$, and the eigenvectors form 
the columns of the matrix $\mathbf{X}$ with $\mathbf{X}^{T} \mathbf{X}=\mathbf{I}$. Define $\mathbf{z}=\mathbf{X}^{T} \mathbf{X}$. The problem in (13) can be written as

$$
\begin{aligned}
\min & \mathbf{z}^{T} \sum \mathbf{z} & =\sum_{i=1}^{K} \Lambda_{i} z_{i}^{2} \\
\text { s.t. } & \mathbf{z}^{T} \mathbf{z} & =1, \quad\left|\mathbf{z}^{T} \mathbf{X}^{T} \mathbf{v}_{1}\right|=\Lambda_{1} \leq \beta .
\end{aligned}
$$

Since $\Lambda_{1}<\Lambda_{2} \leq \cdots \leq \Lambda_{K}$, and $z_{i}^{2} \geq 0$, the optimal solution is $z_{1}=\beta, z_{2}=\sqrt{1-\beta^{2}}$, and $z_{i}=0 \forall i>2$. Therefore, the optimal solution for $\mathrm{x}$ is given by (14).

Corollary 1: With fixed $\epsilon$, the problem in (12) is solved by the following vector:

$$
\mathbf{q}=\frac{\epsilon}{K} \mathbf{1}+\sqrt{K-\frac{\epsilon^{2}}{K}} \mathbf{w}_{2}
$$

where $\mathbf{w}_{2}$ is the Fiedler vector of the modified Laplacian matrix in (9). The problem in (12) has the following solution:

$$
\hat{U}^{n b}=-\frac{\lambda_{2}}{2}
$$

where $\lambda_{2}$ is the Fiedler value of the modified Laplacian matrix in (9).

Proof: The modified Laplacian matrix in (9) is symmetric positive semidefinite with smallest eigenvalue 0 and corresponding eigenvector $\mathbf{1} / \sqrt{K}$. Letting $\mathbf{q}=\sqrt{K} \mathbf{x}$ and $\epsilon=K \beta$, we have the solution in (16). Substituting $\mathbf{q}$ in (12) and letting $\lambda_{i}=\Lambda_{i} \forall i$, we have

$$
\hat{U}^{n b}=-\frac{\frac{\epsilon^{2}}{K^{2}} \lambda_{1}+\frac{K^{2}-\epsilon^{2}}{K} \lambda_{2}}{2(K-\epsilon)}=-\frac{\lambda_{2}(K+\epsilon)}{2 K} .
$$

The minimum is obtained when $\epsilon=0$.

The solution in (17) for the relaxed network bisection connectivity is a lower bound to the real bisection connectivity in (11). If $\lambda_{2}$ can be increased (or equivalently, $-\lambda_{2}$ decreased), then the worst-case bisection connectivity is improved. As mentioned earlier, solving the problem in (11) is $N P$-hard. In the next section, we will develop algorithms to reduce (17) with polynomial-time complexity.

\section{C. k-Connectivity}

In some situations where network robustness is a key issue, it may be desired to guarantee network connectivity in the event that some subsets of the network nodes are disabled. This is the idea behind $k$-connectivity, as subsequently defined.

Definition 4: A graph $G$ with edge set $V(G)$ is said to be $k$-connected if $G \backslash Y$ is connected for all $Y \subseteq V(G)$ with $|Y|<k$. Here, $G \backslash Y$ is the graph that results when the nodes in $Y$ are removed from the graph $G$. In other words, a graph is $k$-connected if it remains connected when fewer than $k$ vertices are deleted from the graph.

If a graph $G$ is $k$-connected, and $k<|V(G)|$, then $k \leq$ $\Delta(G)$, where $\Delta(G)$ is the minimum degree of any vertex $v \in$ $V(G)$. This fact is clear since deleting all neighbors of a vertex of minimum degree will disconnect that vertex from the rest of the graph. Menger's theorem [17], [18], which is a special case of the max-cut min-flow theorem, states how to calculate $k$ for $k$-connectivity.
Theorem 2-(Menger's Theorem): Let $G$ be a finite undirected graph, and let $i$ and $j$ be two nonadjacent vertices. Then, the size of the minimum vertex cut for $i$ and $j$ (the minimum number of vertices whose removal disconnects $i$ and $j$ ) is equal to the maximum number of pair-wise vertex-independent paths from $i$ to $j$. In other words, if $G$ is $k$-connected, then there are at least $k$ pairwise disjoint paths from $i$ to $j$.

The utility function for $k$-connectivity is simply defined to be

$$
U^{k c}=-k
$$

where $k$ represents the maximal value for which the graph is $k$-connected, and where the minus sign is added since we desire smaller values for $U^{k c}$, which is consistent with the other definitions of connectivity. Note that $U^{k c}$ is not related to the link weights. To place a new node in the network to improve $U^{k c}$, one must locate it such that it connects as many nodes as possible and improves the minimum number of links that nodes have to connect to the rest of network.

\section{UAV DePLOYMENT/Movement: ANALYSIS AND ALGORITHMS}

Based on the connectivity definitions formulated in the previous section, we investigate how to optimally deploy a single UAV to improve the network connectivity. We first analyze the two-node one-UAV case in detail. Then, we propose multinode algorithms for UAV deployment and movement. Finally, we discuss some implementation issues.

Recall that the UAV is denoted as node 0 with location $\left(x_{0}, y_{0}\right)$. The UAV deployment problem we consider can be formulated as minimizing one of the network connectivity definitions in (7), (8), (11), or (19) with respect to the UAV's location, i.e.,

$$
\min _{\left(x_{0}, y_{0}\right)} U^{s s}
$$

where the superscript $s s$ is chosen to indicate which of the connectivity definitions is optimized. For the case of global message connectivity, the problem in (20) is equivalent to the well-known Steiner point problem from graph theory [17], [18]. A Steiner point refers to a node added to the network in such a way that the weight or cost of the network's MST is minimized. The Steiner point problem, as well as (20) implemented with the other connectivity definitions, is known to be $N P$-hard [17], [18]. To find approximate solutions with only polynomial complexity, we first analyze the two-node one-UAV case and then propose heuristic approaches in the following sections.

\section{A. Performance Analysis for the Two-Node One-UAV Case}

Suppose two nodes are uniformly distributed within a circle of radius $R$, as shown in Fig. 3. Denote the distance between the two nodes by $d$, so that $0 \leq d \leq 2 R$. Suppose node 1 is located at a distance $r$ from the center. Using standard geometric probability theory [21], it is possible to derive the probability density function (pdf) of the distance between random nodes as a function of $d$ and $r$, as subsequently outlined. Define the 

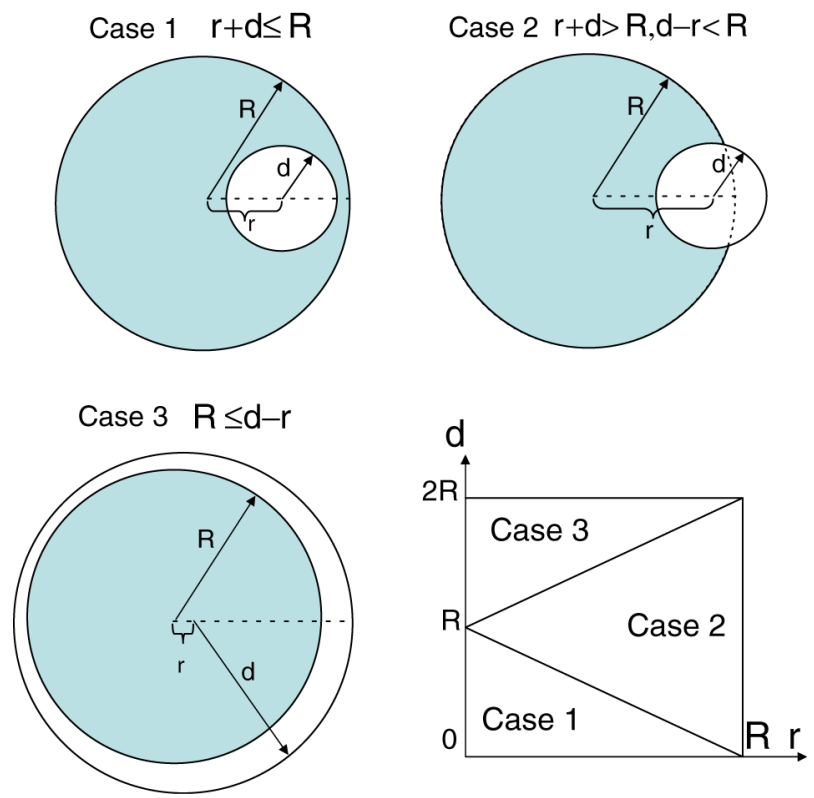

Fig. 3. Two-node analysis.

indicator function $I(r, d) \in\{1,2,3\}$ to represent the following three possible scenarios, as depicted in Fig. 3.

1) $I(r, d)=1$ when $r+d \leq R$.

Because of the uniform distribution, we can calculate the probability that the distance between two nodes is greater than some value $d$ as

$$
P_{r}(d \mid I=1, r)=\frac{\pi R^{2}-\pi d^{2}}{\pi R^{2}}=1-\frac{d^{2}}{R^{2}}
$$

where the numerator is the size of the shaded area, and the denominator is the size of the whole area in Case 1 of Fig. 3.

2) $I(r, d)=2$ when $r+d>R$ and $d-r<R$.

In this case, there are some areas within a distance $d$ to node 1 where node 2 cannot be located, since it would place node 2 outside the radius $R$. An example is shown in Fig. 3. Thus, the probability that the distance between the two nodes is greater than $d$ is proportional to the area of the disk with radius $R$ minus the portion of the disk with radius $d$ that lies within the disk with radius $R$, i.e.,

$P_{r}(d \mid I=2, r)=1-\frac{1}{\pi R^{2}}\left\{d^{2} \arccos \left(\frac{r^{2}+d^{2}-R^{2}}{2 r d}\right)\right.$

$\left.+R^{2} \arccos \left(\frac{r^{2}+R^{2}-d^{2}}{2 r R}\right)-R r \sin \left[\arccos \left(\frac{r^{2}+R^{2}-d^{2}}{2 r R}\right)\right]\right\}$.

The value inside the bracket on the right-hand side of (22) represents the area that is inside both the small circle with radius $d$ and the big circle with radius $R$, as shown in Case 2 of Fig. 3.

3) $I(r, d)=3$ when $d-r \geq R$.

As shown in Case 3 of Fig. 3, for this case, there is no possibility that the second node can be located within the radius $R$, i.e.,

$$
P_{r}(d \mid I=3, r)=0 .
$$

The plot in the lower right corner of Fig. 3 illustrates the relationship between $d$ and $r$ for the preceding three cases and shows that the three cases exhaust all the possible combinations of parameters. Taking the foregoing results together, the cumulative distribution function (cdf) can be calculated by

$$
C D F(d)=1-\int_{0}^{R} P_{r}(d \mid I(d, r), r) \frac{2 \pi r}{\pi R^{2}} d r .
$$

The pdf, which we will denote by $p(d)$, can be obtained by differentiating the cdf function.

The probability that the two nodes can successfully communicate without the UAV relay can be calculated as

$$
P_{r}^{12}=\int_{0}^{2 R} \exp \left(-\frac{\sigma^{2} \gamma(d)^{\alpha}}{C P}\right) p(d) d(d)
$$

where, in this example, we assume symmetric channels $C_{12}=$ $C_{21}=C$, transmit power $P_{1}=P_{2}=P$, and interference $\sigma_{1}^{2}=$ $\sigma_{2}^{2}=\sigma^{2}$. By the triangle inequality, it is clear that if a UAV is to be added to the link, it must be located on the line between node 1 and node 2 . Assuming symmetry between all links, from (4), we have

$$
\begin{aligned}
P_{r}^{12} & =P_{r}^{10} P_{r}^{02} \\
& =\exp \left(-\frac{\sigma^{2} \gamma\left[D_{10}^{\alpha}+\left(D_{12}-D_{10}\right)^{\alpha}\right]}{C P}\right) .
\end{aligned}
$$

By differentiating $P_{r}^{12}$ with respect to $D_{10}$, the optimal UAV location is found to be at the midpoint between the two nodes if $\alpha$ is no less than 2. With the UAV in this position, the probability of "global" message connectivity is given by

$$
P_{r}^{12}=\int_{0}^{2 R} \exp \left(-\frac{2 \sigma^{2} \gamma\left(\frac{d}{2}\right)^{\alpha}}{C P}\right) p(d) d(d) .
$$

The two-node one-UAV case represents a scenario where it is critical to maintain a given link between two mobile nodes, and the preceding analytical result quantifies how much a UAV can improve the connectivity of the link. We set up a simulation to test the analysis results. Two nodes are randomly and uniformly located within a cell of radius $R$. The transmission power is $300 \mathrm{dBm}$, the noise power is $\sigma^{2}=10^{-7} \mathrm{dBm}$, the SNR requirement is given by $\gamma=10 \mathrm{~dB}$, and the propagation loss factor is $\alpha=3$. In Fig. 4, we show the global message connectivity for different values of the radius $R$. We list the numerical and analytical results for the no-UAV two-node case and one-UAV two-node case, respectively. We see that the analysis exactly matches the numerical results. The larger the cell size, the more improvement a UAV relay can provide. At a cell radius of $1000 \mathrm{~m}$, the improvement with one UAV is as much as $240 \%$.

\section{B. Optimal UAV Deployment}

In this section, we consider the problem of UAV deployment [determining $\left(x_{0}, y_{0}\right)$ ] to improve global message connectivity, 


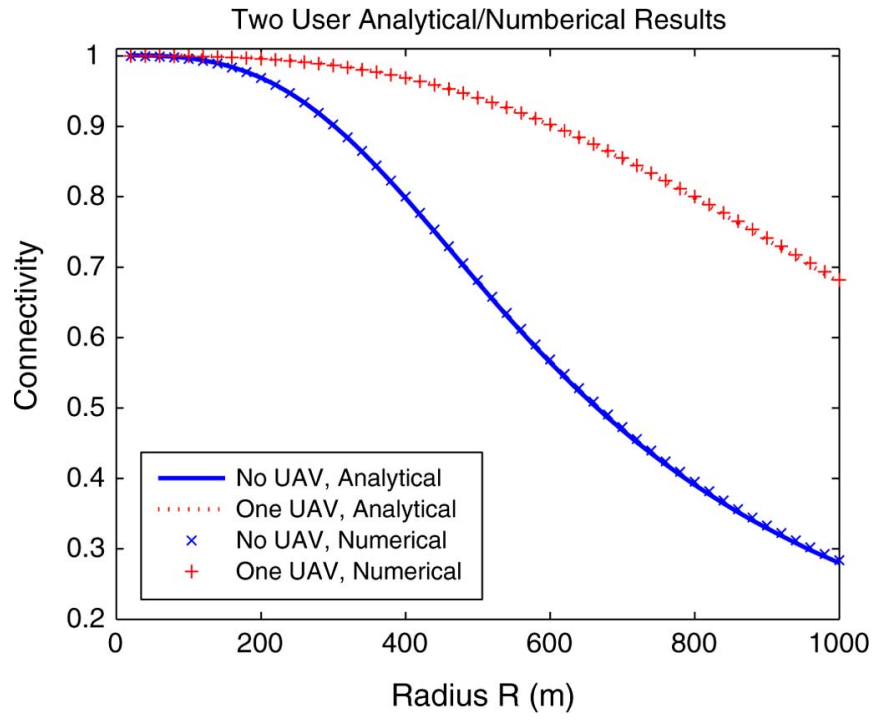

Fig. 4. Two-node one-UAV analytical and numerical results for global message connectivity.

worst-case connectivity, and network bisection connectivity. While, for simplicity, we consider optimization of the UAV position in only two dimensions, it is straightforward to extend the algorithms to the cases involving altitude as a third dimension. In general, the problem is $N P$-hard, but we propose an adaptive algorithm to find a local optimum with reasonable complexity. Specifically, starting from any initialization point, we determine how to change the UAV's location in its neighborhood so that a better MST can be obtained. For global message connectivity and worst-case connectivity, the gradients for such a search can be written as

$$
g_{0}=\frac{d U\left(x_{0}, y_{0}\right)}{d x_{0}}+z \frac{d U\left(x_{0}, y_{0}\right)}{d y_{0}} .
$$

A linear search algorithm [22] is utilized to reduce the complexity of the gradient method. The stopping criteria is chosen to be $\left\|g_{0}\right\|^{2} \leq \varepsilon$, where $\varepsilon$ is a small positive number, or where the Karush-Kuhn-Tucker (KKT) condition holds [22], i.e., at which the local optimum is achieved.

For network bisection connectivity, we direct the UAV to a location that maximizes the Fiedler value of the modified Laplacian matrix $\mathbf{L}$ in (9). The existence of the derivative of the spectral radius is provided by the following theorem [24].

Theorem 3: Let $\lambda$ be a simple eigenvalue of symmetric matrix $\mathbf{B}$ with eigenvector $\mathbf{v}$. Let $\tilde{\mathbf{B}}=\mathbf{B}+\Delta \mathbf{B}$, where $\Delta \mathbf{B}$ is a small perturbation. Then, there exists a unique eigenvalue $\tilde{\lambda}$ of matrix $\tilde{\mathbf{B}}$ such that

$$
\tilde{\lambda}=\lambda+\frac{\mathbf{v}^{H} \Delta \mathbf{B} \mathbf{v}}{\mathbf{v}^{H} \mathbf{v}}+\mathrm{O}\left(\|\Delta \mathbf{B}\|^{2}\right) .
$$

Thus, the gradient of the Fiedler value is given by

$$
g_{0}=\frac{\mathbf{w}_{2}^{H}\left[d \mathbf{L}\left(x_{0}, y_{0}\right)\right] \mathbf{w}_{2}}{\mathbf{w}_{2}^{H} \mathbf{w}_{2}}+z \frac{\mathbf{w}_{2}^{H}\left[d \mathbf{L}\left(x_{0}, y_{0}\right)\right] \mathbf{w}_{2}}{\mathbf{w}_{2}^{H} \mathbf{w}_{2}}
$$

where $\mathbf{w}_{2}$ is the Fiedler vector.
The general optimization problem in (20) can have many local minima, as shown in the following simple example. Suppose there are only three nodes and one UAV in the network. The three nodes are located on a line with coordinates $(0,0),(1,0)$, and $(1.8,0)$. Some simple calculations reveal that for global message and worst-case connectivity, there are two optimal locations for the UAV: $(0.5,0)$ and $(1.4,0)$. To overcome the local optimum problem, we propose the following two initialization methods.

1) Random Initialization: This approach generates a number of seeds within the area of the MANET and lets the gradient method find the local optima. The location with the smallest value among all the local optima is selected as the initialization. The advantage of this initialization method is that the global optimum can be obtained with a high probability when the density of the initialization seeds is sufficiently high. The disadvantage is that the computational complexity is high, particularly when the number of nodes is large.

2) Heuristic Initialization: Suppose that the MST without the UAV is constructed, and that the maximal link weight occurs between node $i$ and node $j$. The heuristic initialization point for the UAV is at the midpoint of these two nodes, i.e.,

$$
x_{0}^{0}=\frac{x_{i}+x_{j}}{2} \quad y_{0}^{0}=\frac{y_{i}+y_{j}}{2} .
$$

The rationale is to improve the worst-case link and make the initial performance improvement as large as possible before applying the gradient method.

3) Weighted Centroid Initialization: For this case, the UAV is initialized to be at the weighted center of mass of the network $x_{0}=\sum_{i=1}^{K} \alpha_{i} x_{i}$ and $y_{0}=\sum_{i=1}^{K} \alpha_{i} y_{i}$, where the node weights are normalized to satisfy $\sum_{i=1}^{K} \alpha_{i}=1$. The preceding heuristic initialization is a special case of this more general approach, where the two nodes with the worst link are assigned weights of $1 / 2$. Other possible choices include uniform weighting over the entire network $\left(\alpha_{i}=(1 / K)\right)$ or over some carefully chosen subset (e.g., nodes that are weakly connected to the network), weights that are inversely proportional to the degree of each node, etc.

The overall algorithm for finding the best location to deploy the UAV is summarized in Table I. This algorithm is applicable to improving any of the three types of connectivity: global message, worst case, and network bisection. The complexity of the algorithm for each iteration is $O\left(K^{2}\right)$ due to the calculation of the MST. Note that the complexities of the gradient calculation and line search are independent of $K$. Since $U$ at each iteration of the algorithm is nonincreasing and the solution has a lower bound, the algorithm always converges.

\section{C. $k$-Connectivity Improvement Using DT}

In this section, we study the UAV deployment problem for $k$-connectivity. The gradient method is not applicable in this 
TABLE I

UAV Deployment Algorithm for Global Message, Worst Case, and Network Bisection Connectivity Optimization

\begin{tabular}{|l|l|}
\hline Initialization & $\begin{array}{l}\text { Initialize UAV location }\left(x_{0}^{0}, y_{0}^{0}\right) \text { using either heuristic } \\
\text { or random methods; Set } t=0 .\end{array}$ \\
\hline Iteration & $\begin{array}{l}\text { 1. Calculate gradient } g_{0}^{t} \text { in }(28) . \\
\text { 2. Update step index: } t=t+1 . \\
\text { 3. Line search over gradient direction with } \zeta>0 \text { so that } U \text { is } \\
\text { optimized, i.e., } x_{0}^{t}+z y_{0}^{t}=x_{0}^{t-1}+z y_{0}^{t-1}-\zeta g_{0}^{t} .\end{array}$ \\
\hline Stopping criteria & Gradient is sufficiently small $\left(\left\|g_{0}\right\|^{2} \leq \varepsilon\right)$ or KKT condition holds. \\
\hline Return UAV location $\left(x_{0}, y_{0}\right)$ & If random initialization, select the best achieved local optimum. \\
\hline
\end{tabular}

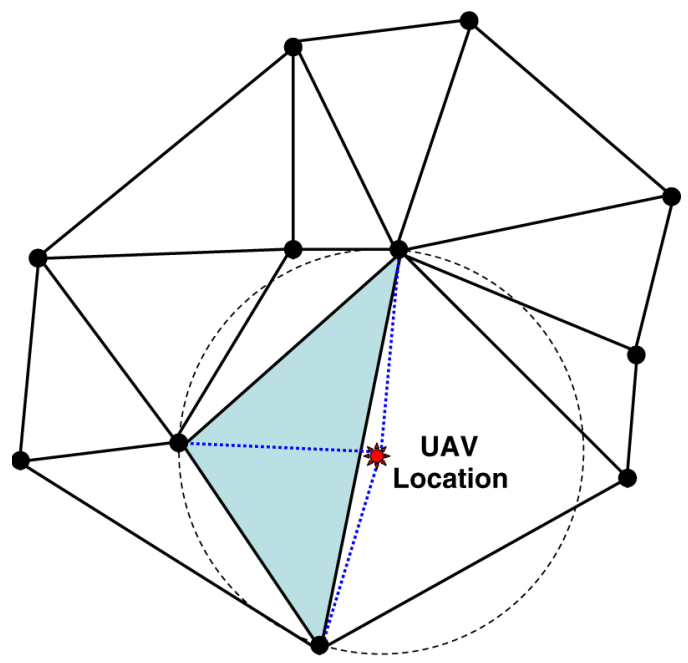

Fig. 5. DT and UAV deployment.

case, since $k$-connectivity is integer valued and, hence, nondifferentiable. Instead, we propose a heuristic algorithm based on DT [17], [18], which is defined below. Note that for our application, a triangulation is defined as the subdivision of the area covered by a network into nonoverlapping triangles, where the vertices of the triangles are nodes in the network. One such example is shown in Fig. 5.

Definition 5: A DT over a network of nodes has the property that none of the triangles has a circumcircle that contains a node.

There are certain situations where the DT does not exist or is not unique, such as when three nodes exactly lie on a line, or a group of four or more nodes exactly lie on a circle. We ignore such scenarios in our application, since they will occur with probability zero. ${ }^{2}$ Under this assumption, the DT can be calculated by the divide-and-conquer algorithm [17], [18] with $O(K \log K)$ complexity.

We propose a greedy algorithm to improve $k$-connectivity based on DT. First, we identify all of the Delaunay triangles

\footnotetext{
${ }^{2}$ For the three-user case, there always exists a Delaunay triangle. However, for any additional user, this user's location has to be located exactly on the circle shown in Fig. 5 to satisfy the Delaunay triangulation. This causes the probability of zero.
}

that possess at least one node of the smallest degree among all the nodes in the network. Then, we place a candidate UAV at the circumcenter of each of these triangles, as shown in Fig. 5 (the blue triangle is the one whose circumcircle is drawn). The circumcenter is the point that is equidistant to the vertices of the Delaunay triangle. With very high probability, the UAV will be able to connect with at least one of the nodes in the triangle and, in many instances, with the other nodes that are part of or even outside the triangle. As a result, we expect that the degree of several nodes will typically be increased. Finally, we select the candidate UAV location that provides the largest improvement in $k$-connectivity. If there is a draw, then the UAV with more links is selected. If there is still a draw, then one of the resulting locations is selected at random. The complete algorithm for $k$ connectivity improvement is found in Table II.

\section{Optimal UAV Movement}

In this section, we assume that the initial UAV deployment has been made as previously described, i.e., $\left(x_{0}, y_{0}\right)$ is known. We try to determine the movement of the UAV so that the best possible network connectivity is maintained as the network topology changes. We assume that $S_{0}$ is the speed and direction of the UAV, as in (1). From (3), (4), and (6), the gradient for a change in utility function can be written as

$$
\frac{d U}{d t}=\frac{d}{d t}\left(U\left(D_{i j}^{*}\right)-U\left(D_{i j}\right)\right)
$$

where $\quad D_{i j}^{*}=\left\|x_{i}+z y_{i}+S_{i} d t-x_{j}-z y_{j}-S_{j} d t\right\|^{2} . \quad$ The UAV movement problem formulation is given by

$$
\begin{gathered}
\max _{S_{0}} \lim _{d t \rightarrow 0}-\frac{d U}{d t} \\
\text { s.t. } v_{\min }^{2} \leq\left\|S_{0}\right\|^{2} \leq v_{\max }^{2}
\end{gathered}
$$

where $v_{\min }$ and $v_{\max }$ are the minimum and maximum speeds of the UAV, respectively. Notice that zero speed means that the $\mathrm{UAV}$ is hovering.

Since the UAV is airborne, we need to consider the issue of speed constraints. If the gradient in (32) is small, then the connected nodes are hardly moving, and hence, the UAV does not change position. Under this condition, the UAV must fly 
TABLE II

$k$-CONNECTIVITY IMPROVEMENT ALGORITHM USING DT

\begin{tabular}{|l|}
\hline 1. Construct the Delaunay triangulation. \\
\hline 2. Select the triangles that possess at least one node with smallest degree. \\
\hline 3. Place a candidate UAV at the circumcenter of the triangles in the selected set. \\
\hline 4. For each candidate UAV, find all new network connections that result from addition of \\
the UAV, i.e., all new links whose probability of successful transmission is larger than $\delta$. \\
\hline 5. Select the candidate UAV location that results in the largest increase in $k$-connectivity. \\
If there is a draw, select the UAV location that will possess the most links. \\
\hline
\end{tabular}

TABLE III

ALGORITHM FOR OPTIMAL UAV MOVEMENT

\begin{tabular}{|l|l|}
\hline Monitor & Measure $\left(x_{i}, y_{i}\right)$ and $S_{i}$ for $i \in \mathbf{V}(G)$. \\
\hline Movement & Circle: $\quad$ If $\left|\mu \frac{d U}{d t}\right|<v_{\text {min }}$. \\
& Fly: $\quad$ If $\left|\mu \frac{d U}{d t}\right| \geq v_{\text {min }}$ \\
$\qquad S_{i}= \begin{cases}-\mu \frac{d U}{d t}, & \text { if }\left|\mu \frac{d U}{d t}\right| \leq v_{\max } \\
-v_{\max }\left|\frac{d U}{d t} \frac{d U}{d t}\right| & \text { otherwise. }\end{cases}$ \\
\hline Update & Perform algorithm in Table I to check for improved deployment. \\
\hline
\end{tabular}

in a small circle. When the gradient is large enough, the UAV flies against the gradient direction with speed proportional to the magnitude of the gradient. When the gradient is too large, the UAV can only fly in the direction of the gradient with its maximum speed $v_{\max }$. The algorithm for calculating the velocity of the UAV $S_{0}$ is given in Table III, where $\mu$ is a constant that can experimentally be determined.

We assume that within a short period of time $d t$, the network topology does not significantly change. The movement of the UAV will only affect the weights of the links connected to it, and it only needs to monitor nearby nodes as it updates its position. As such, the complexity and signaling overhead is greatly reduced. The two algorithms in Tables I and III can be implemented in turn. First, the deployment algorithm is used to find the best location the UAV initially flies to. Then, the movement algorithm keeps track of the mobility of the distributed nodes, and small adjustments are made to the UAV location. Periodically, the deployment algorithm can be reapplied to see if a new global optimum has emerged. The frequency with which the deployment algorithm is reapplied depends on the degree of node mobility.

\section{E. Challenges and Limitations}

The most significant challenge to the algorithms previously described is the requirement that some centralized locations (the one that computes the optimal UAV position and velocity, which is not necessarily the UAV itself) must possess or be able to estimate the position and speed of all the network nodes as well as the quality (in terms of successful transmission probabilities) of all the network links. Link quality is constantly monitored, since it determines the modulation and coding schemes that are employed. Since the physical network topology changes relatively slowly, and since the UAV cannot instantaneously relocate itself, it makes sense to only consider the link quality averaged over several independent fades. All things considered, the update rate for the UAV location can be on the order of at least several seconds. If the network is not too large, it is reasonable to assume that GPS (if available) and link quality information could be shared among all nodes over such an interval without significantly impacting the network throughput given that the network is connected. In a network with a very large number of nodes, it is unlikely that a single UAV would be used to provide or improve connectivity. In such cases, a more likely scenario would involve the UAV maintaining connectivity with a selected set of nodes that provide a backbone for network communications. In the absence of GPS, other node or network-localization techniques have been proposed [25] to obtain position information.

The preceding arguments apply to computational complexity as well. While the speed and heading of the UAV relay are adjusted on the order of every few seconds, this computation only involves the relatively few nodes that are connected to the UAV. The more complicated deployment algorithm, which involves processing data from all network nodes, is done even more infrequently, perhaps on the order of minutes. The proposed gradient-based search technique is easily amenable to adaptive implementation and can constantly be running in the background, in between maneuvers, so that the solution is immediately available at the time of redeployment.

There are a number of limitations to the connectivity definitions and UAV deployment strategies previously outlined that bear mentioning. First, we have not taken energy constraints into account. Even if two nodes are equally well connected to the network in terms of link quality, the network may benefit most by providing relay coverage to one over the other if it has 
Successful probability as function of AUV location

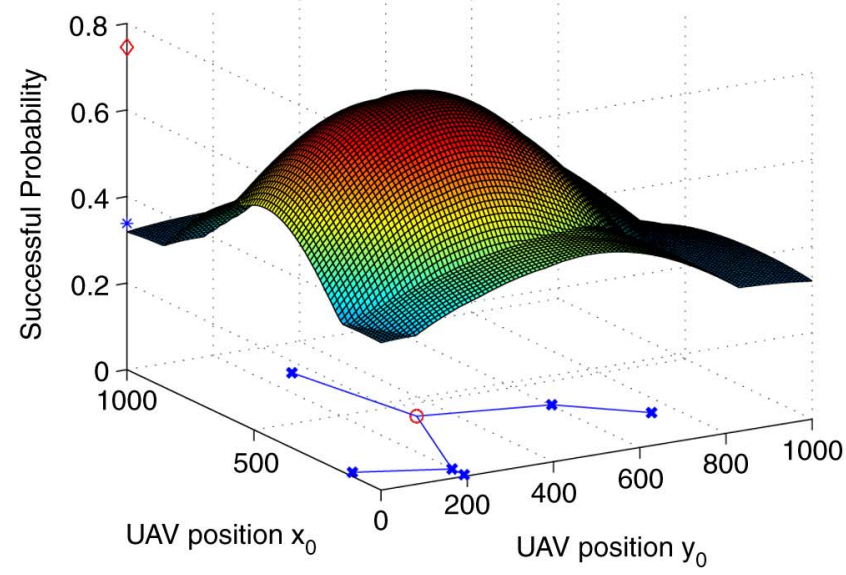

(a)

$\lambda_{2}-$ connectivity as function of AUV location

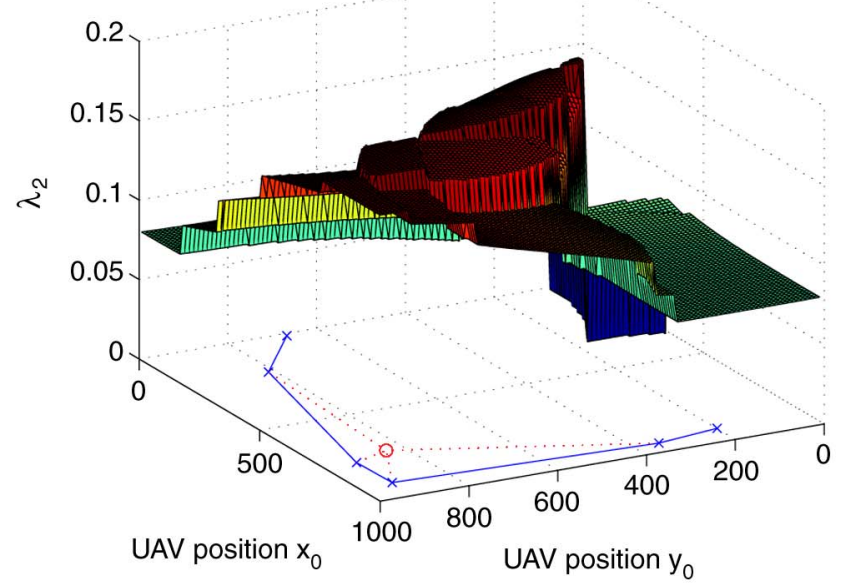

(c)
Successful probability as function of AUV location

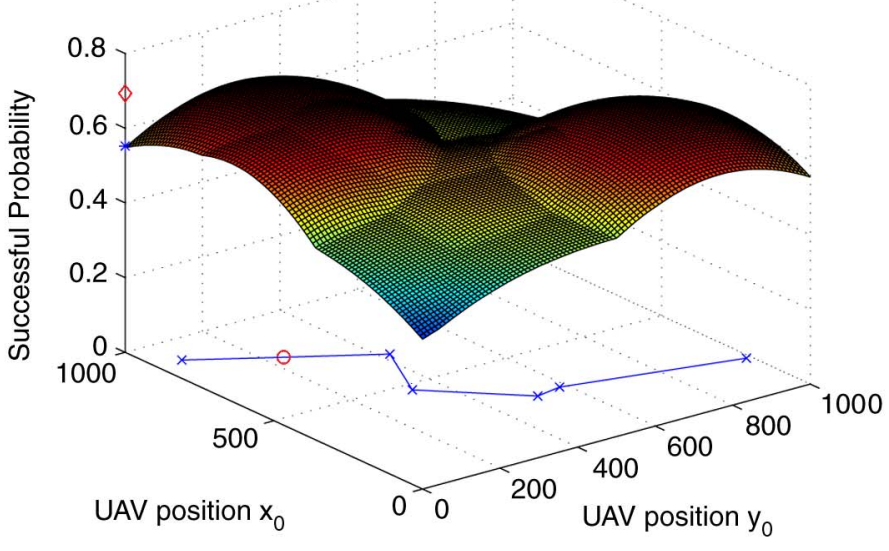

(b)

k-connectivity as function of AUV location

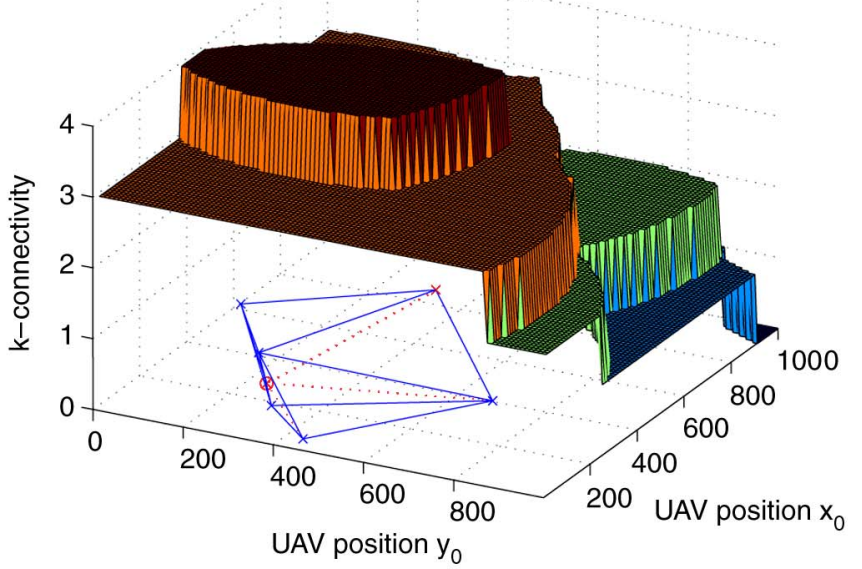

(d)

Fig. 6. Connectivity as a function of UAV location. (a) Global message connectivity, $K=6$. (b) Worst-case connectivity, $K=6$. (c) Network bisection connectivity, $K=6$. (d) $k$-connectivity, $K=6$.

nearly depleted its available energy. However, it is not difficult to envision extensions of the preceding work that modify the edge weights to take both link quality and energy reserves into account. Second, the links between the two UAVs are indeed different. However, they experience independent fading, which is identical to Rayleigh fading due to the same distance. As a result, the statistics are similar, the symmetric links between pairs of nodes are assumed, and the graph is unidirectional. Networks composed of heterogeneous nodes or links with asymmetric uplink and downlink capabilities will lead to a directed graph. The link qualities of asymmetric graphs can be obtained by practical measurement. Such cases are beyond the scope of this paper but provide interesting avenues for future research. Third, beyond the Rayleigh distribution used in the system model, other models can also be employed in a similar way. In practice, the distribution of the channels can be collected and employed to the framework of this paper in a straightforward way. Fourth, in practice, the global information might be hard to obtain. In the proposed algorithms, the UAV tries to improve the performance around the places where there is a weakest link or on the boundary where the network is divided. As a result, only the information about the nodes around is necessary. Moreover, the UAV mostly serves for the backbone of the network. This backbone may be composed of a relatively few key communications nodes, and it is more reasonable to assume that the position and channel condition for these nodes could be known. Note that such global information is only needed relatively infrequently on the order of minutes. Consequently, the practical implementation can be simplified. Finally, the preceding algorithms address the deployment and motion of a single UAV. Obviously, improved network performance can be achieved by deploying multiple airborne relays of this type but at the expense of the need for a multidimensional optimization. The techniques proposed in this paper could be used to deploy multiple UAVs in a sequential manner, i.e., by treating the previously deployed UAVs as fixed network nodes and accordingly optimizing the position of the next UAV, but such an approach is clearly suboptimal in a global sense. This is a problem that would also be a fruitful area of further research. 


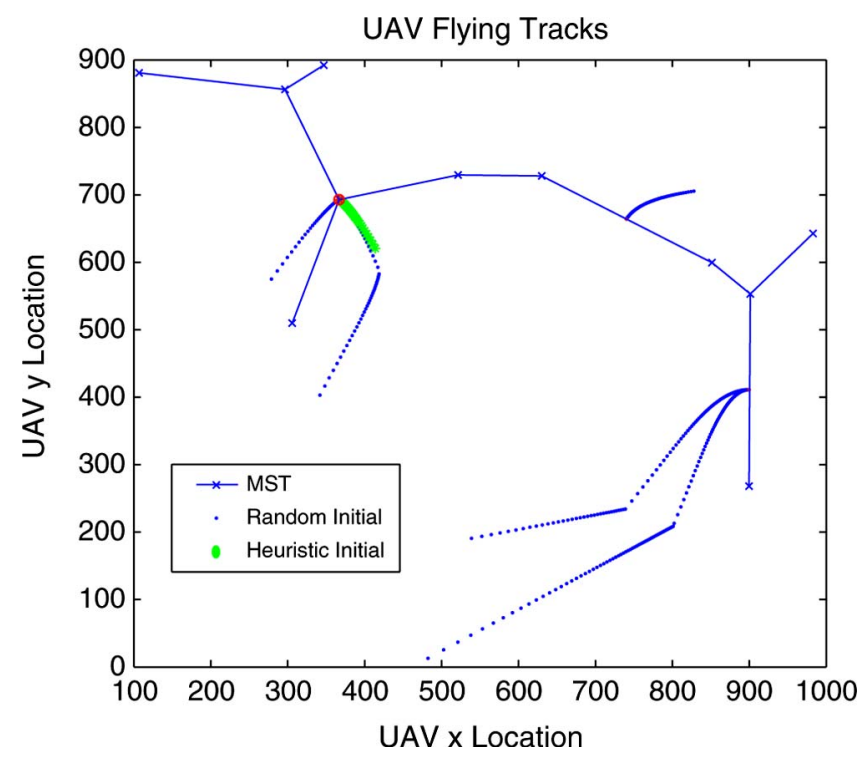

Fig. 7. UAV trajectories with different initializations. $K=10$.

\section{Simulations}

To demonstrate the effectiveness of the proposed algorithms, we use the following simulation study: A total of $K$ nodes are randomly located within a square region of $1000 \times 1000 \mathrm{~m}$. The transmission power for each node is $300 \mathrm{dBm}$, the received interference plus noise power is $\sigma^{2}=10^{-7} \mathrm{dBm}$, the SNR requirement is given by $\gamma=10 \mathrm{~dB}$, and the propagation loss factor is $\alpha=3$. Without loss of generality, for the communication links between different mobile nodes, we assume $C_{i j}=C_{1}$ $\forall i, j \in\{1,2, \ldots, K\}$; for the communication link between the $\mathrm{UAV}$ and the mobile nodes, $C_{i j}=C_{0}, i$ or $j=0$. For the simulations conducted here, we assume that $C_{0}=2$ and $C_{1}=1$.

In Fig. 6(a), we show a snapshot of the global message connectivity as a function of the UAV location $\left(x_{0}, y_{0}\right)$. On the $Z$-axis, we show the connectivity of the network without the $\mathrm{UAV}$ as a star with a value of 0.3379 . By deploying the UAV at the best location $\left(x_{0}^{*}, y_{0}^{*}\right)=(330,420)$, the connectivity probability is improved to 0.7452 , as shown by a diamond on the $Z$-axis. On the $x-y$ plane, we show the MST with the nodes denoted by crosses and the UAV denoted by a circle. We see that in this case the UAV positions itself to improve multiple links. Moreover, from the curve, we can see that there are many local optima for $\left(x_{0}, y_{0}\right)$. In Fig. 6(b), we show the worst-case connectivity, where the UAV improves the worst link in the network.

In Fig. 6(c), we show the Fiedler value $\lambda_{2}$ as a function of the UAV location. We can see that the nodes are divided into three clusters. By placing the UAV at the best location $\left(x_{0}^{*}, y_{0}^{*}\right)=$ $(840,730)$, the UAV can connect to all three clusters so that the cost to divide the whole network increases. In Fig. 6(d), we show $k$-connectivity as a function of UAV location. Within a certain range of positions, the UAV can improve the $k$-connectivity by one, since it can connect to all the other nodes.

In Fig. 7, we show sample UAV trajectories for different types of initialization using the global message connectivity as the performance metric. For the case of random initialization, the results are shown for five different initial seeds. While the UAV converges to a common location in most cases, in general, we see that different initializations lead to different local optima. We also observe that the trajectories are not smooth and that the UAV may change directions. This is because the derivative of $U$ is not continuous, which can easily be observed from Fig. 6(a).

In the following examples, we illustrate the results of several Monte Carlo simulations, where the results are obtained by averaging over 500 different network realizations. In Fig. 8(a) and (b), we show the global message and the worst-case connectivity, respectively, for different numbers of nodes. For both cases, we show the performance of no UAV and a single UAV with both random and heuristic initializations. As a performance benchmark, to ensure that we find the global optimum for comparison purposes, the random initialization method was implemented with 2500 different initialization points. We can see that performance drops at first when the number of nodes increases from a small number, since we assume a connected network, and the probability that one of the network links will cover a long distance initially increases. Since the network dimensions are bounded, continuing to add nodes increases the node density to the point that this probability begins to decrease. We see that the addition of a single UAV improves the connectivity of the network by $109 \%$ when the number of nodes is 4 . The degree of improvement shrinks as the number of nodes increases, since a higher node density means that most of the links already have good connection probabilities, and the addition of the UAV only offers a slight improvement. For worst-case connectivity, the UAV improves performance by up to $60 \%$. The heuristic initialization only has slightly worse performance compared with the best of 2500 random initializations, indicating that convergence to a local rather than global optimum is not a significant problem in this case.

In Fig. 9(a) and (b), we show the network bisection connectivity and the $k$-connectivity as functions of minimal link success probability $\delta$. Recall that the link from node $i$ to node $j$ is connected if the probability of a successful transmission satisfies $P_{i j} \geq \delta$. When $\delta$ increases, both types of connectivity are reduced, because there are fewer available connected links between the nodes. When the number of nodes is large, the connectivity is also improved since the average distance between nodes is reduced. The numerical values of the network bisection connectivity with and without the UAV are also shown. The improvement is about $0.5-1$ for the second largest eigenvalue $\lambda_{2}$, and consequently, the actual cost to cut the network in half increases. For $k$-connectivity in Fig. 9(b), the UAV can improve $k$ by about 1 in all cases. However, multiple UAVs are required in situations where the network has well-separated areas of weak connectivity.

In Fig. 10, we show the average UAV speed and the probability that the UAV falls into a local optimum for global message connectivity for a case where $K=5$. In this example, node mobility is governed by the random direction model. All nodes move in arbitrary directions with speeds uniformly distributed from zero to the maximum speed $v_{\max }=30 \mathrm{~m} / \mathrm{s}$. The direction is uniformly distributed from 0 to $2 \pi$. The UAV updates its direction every $10 \mathrm{~s}$, and the total time for each 


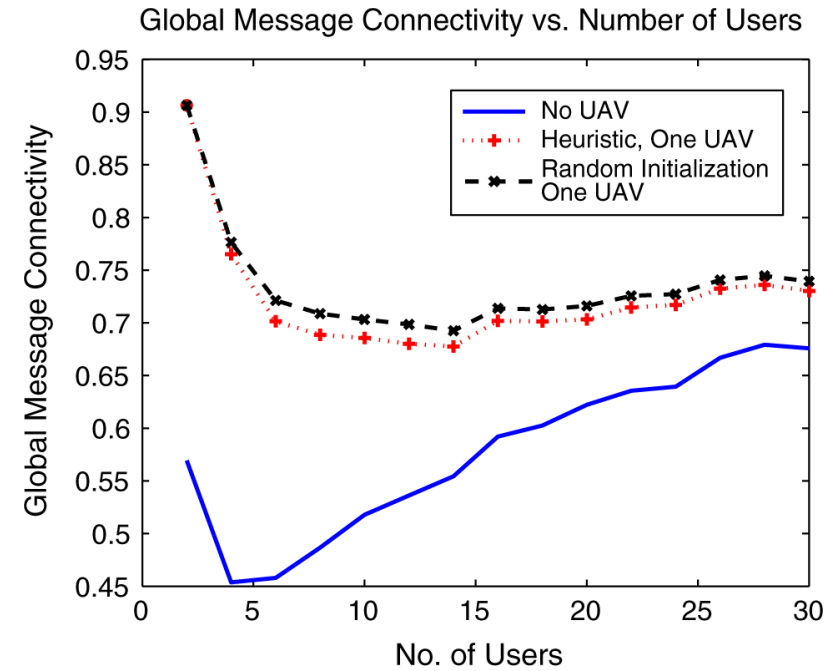

(a)

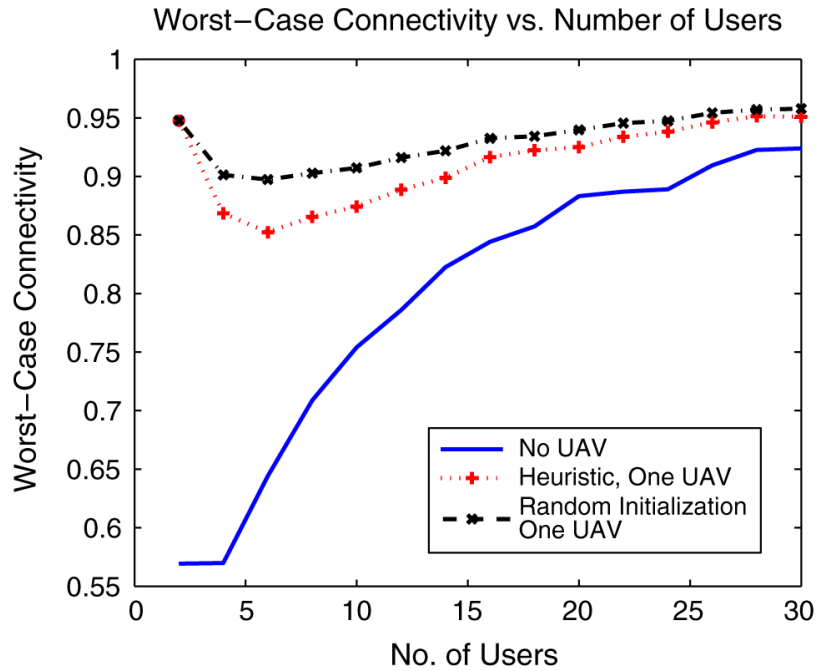

(b)

Fig. 8. Global message connectivity and worst-case connectivity versus number of nodes. (a) Global message connectivity. (b) Worst-case connectivity.

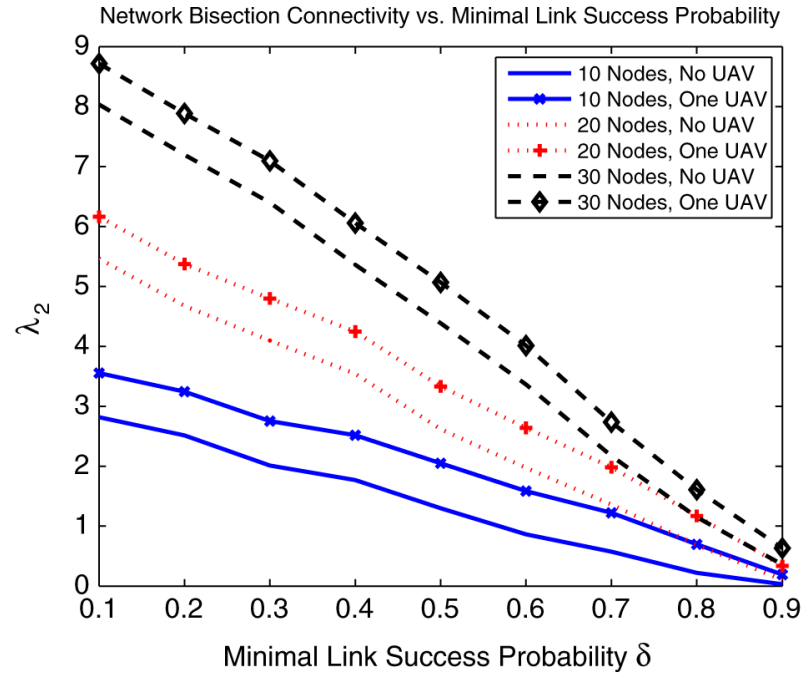

(a)

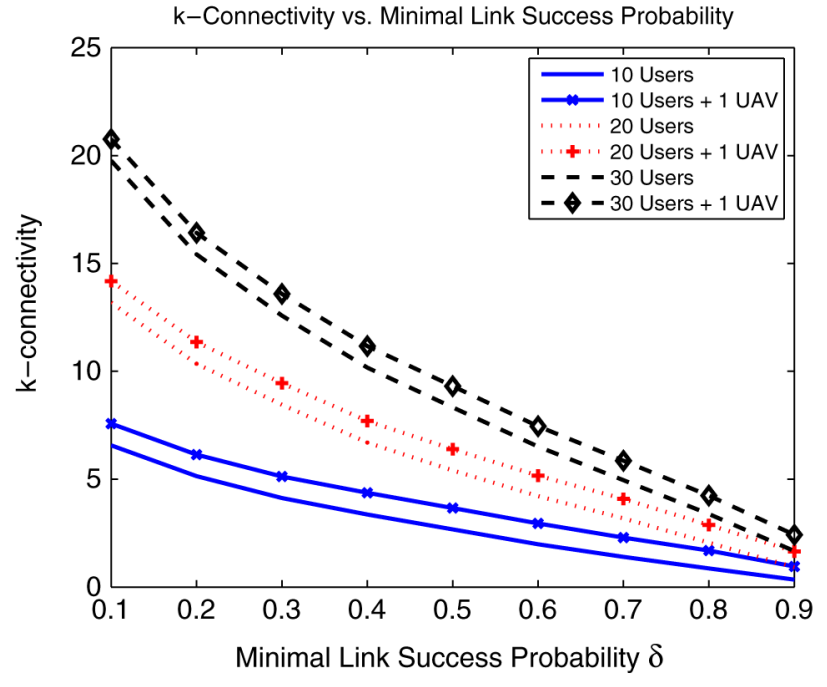

(b)

Fig. 9. Connectivity as a function of minimal link success probability. (a) Network bisection connectivity versus $\delta$. (b) $k$-connectivity versus $\delta$.

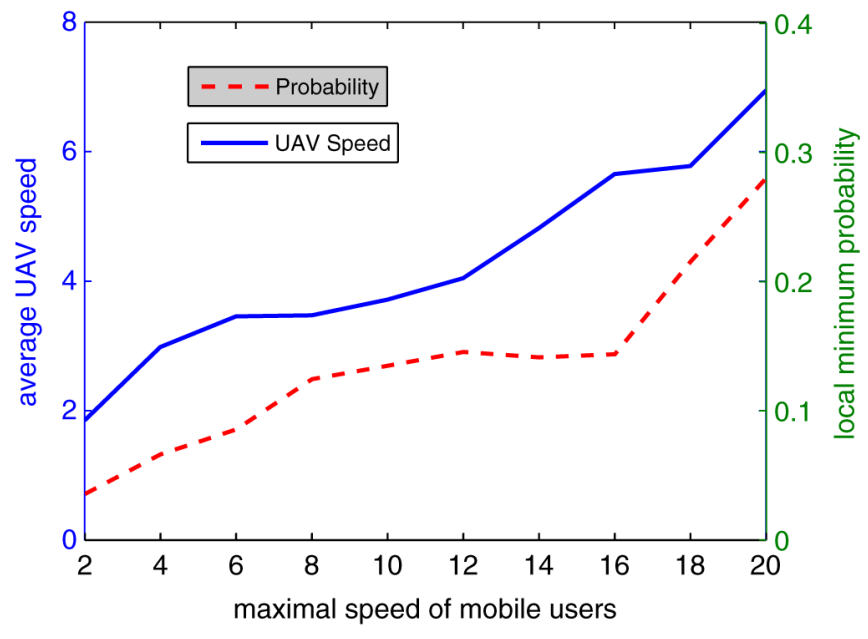

Fig. 10. UAV speed and probability of local optima versus mobility. network simulation is $300 \mathrm{~s}$. We can see that the average speed of the UAV increases according to the nodes' mobility. The probability that the UAV falls into a local optimum also increases with the speed of the mobile nodes. Clearly, in cases of high mobility, the frequency with which one should use the deployment algorithm in Table I will be higher. It is also worth mentioning that the UAV movement for $k$-connectivity is different than for the other connectivity definitions. From the flat curve in Fig. 6(d), we see that the $k$ value is relatively stable if the nodes are moving. A reasonable approach in this case would be that, after initialization, when the UAV detects a reduction in $k$, it applies the algorithm in Table II to find a better position to which to relocate.

\section{CONCLUSION}

In this paper, we have studied how to utilize UAVs to improve the network connectivity of a MANET. We defined four types 
of connectivity: global message, worst case, network bisection, and $k$-connectivity. We formulated deployment and movement problems for the UAV and developed adaptive algorithms to provide a simple solution as well as good performance. We provided a theoretical analysis for a simple two-node one-UAV case and demonstrated that the addition of the UAV provided an improvement in global message connectivity of $240 \%$. For general network setups, a UAV can improve the global message connectivity and the worst-case connectivity by $109 \%$ and $60 \%$, respectively. We showed that network bisection and $k$-connectivity are also improved by the addition of a UAV to the network.

\section{REFERENCES}

[1] R. Beard, T. McLain, D. Nelson, and D. Kingston, "Decentralized cooperative aerial surveillance using fixed-wing miniature UAVs," Proc. IEEE-Special Issue Multi-Robot Syst., vol. 94, no. 7, pp. 1306-1324, Jul. 2006, Tech. Rep. [Online]. Available: https://dspace.byu.edu/handle/ $1877 / 60$

[2] R. Beard, D. Kingston, M. Quigley, D. Snyder, R. Christiansen, W. Johnson, T. Mclain, and M. Goodrich, "Autonomous vehicle technologies for small fixed wing UAVs," AIAA J. Aerosp. Comput., Inf., Commun., vol. 2, no. 1, pp. 92-108, Jan. 2005.

[3] D. W. Casbeer, D. B. Kingston, R. W. Beard, T. W. McLain, S. Li, and R. Mehra, "Cooperative forest fire surveillance using a team of small unmanned air vehicles," Int. J. Syst. Sci., vol. 37, no. 6, pp. 351360, May 2006, Tech. Rep. [Online]: Available: https://dspace.byu. edu/handle/1877/55.

[4] D. L. Gu, G. Pei, H. Ly, M. Gerla, B. Zhang, and X. Hong, "UAV aided intelligent routing for ad hoc wireless network in single area theater," in Proc. IEEE Wireless Commun. Netw. Conf., Chicago, IL, Sep. 2000, vol. 3 , pp. 1220-1225.

[5] D. L. Gu, H. Ly, X. Hong, M. Gerla, G. Pei, and Y. Lee, "C-ICAMA, a centralized intelligent channel assigned multiple access for multi-layer ad-hoc wireless networks with UAVs," in Proc. IEEE Wireless Commun. Netw. Conf., Chicago, IL, Sep. 2000, vol. 2, pp. 879-884.

[6] K. Xu, X. Hong, M. Gerla, H. Ly, and D. L. Gu, "Landmark routing in large wireless battlefield networks using UAVs," in Proc. IEEE MILCOM, Washington, DC, Oct. 2001, vol. 1, pp. 230-234.

[7] I. Rubin, A. Behzad, H. Ju, R. Zhang, X. Huang, Y. Liu, and R. Khalaf, "Ad hoc wireless networks with mobile backbones," in Proc. 15th IEEE Int. Conf. Pers., Indoor Mobile Radio Commun., Barcelona, Spain, Sep. 2004, vol. 1, pp. 566-573.

[8] O. Dousse, P. Thiran, and M. Hasler, "Connectivity in ad-hoc and hybrid networks," in Proc. INFOCOM, New York, Jun. 2002, pp. 1079-1088.

[9] P. Gupta and P. R. Kumar, "Critical power for asymptotic connectivity," in Proc. IEEE Conf. Decision Control, Tampa, FL, Dec. 1998, pp. 1106-1110.

[10] L. E. Miller, "Probability of a two-hop connection in a random mobile network," in Proc. 35th Conf. Inf. Sci. Syst., Baltimore, MD, Mar. 2001.

[11] P. Santi and D. M. Blough, "The critical transmitting range for connectivity in sparse wireless ad hoc networks," IEEE Trans. Mobile Comput., vol. 2, no. 1, pp. 25-39, Jan.-Mar. 2003.

[12] F. Xue and P. R. Kumar, "The number of neighbors needed for connectivity of wireless networks," ACM J. Wireless Netw., vol. 10, no. 2, pp. 169-181, Mar. 2004.

[13] N. Li and J. C. Hou, "Improving connectivity of wireless ad hoc networks," in Proc. 2nd Annu. Int. Conf. MobiQuitous, San Diego, CA, Jul. 2005, pp. 314-324.

[14] S. Khuller, "Approximation algorithms for finding highly connected subgraphs," in Approximation Algorithms for NP-Hard Problems, D. S. Hochbaum, Ed. Boston, MA: PWS, 1996.

[15] C. Bettstetter, "On the minimum node degree and connectivity of a wireless multihop network," in Proc. ACM Int. Symp. MobiHoc, Lausanne, Switzerland, Jun. 2002, pp. 80-91.

[16] H. Zhang and J. C. Hou, "On the critical total power for asymptotic k-connectivity in wireless networks," in Proc. IEEE INFOCOM, Miami, FL, Mar. 2005, pp. 466-476.

[17] R. Diestel, "Graph theory," in Graduate Texts in Mathematics, 3rd ed., vol. 173. New York: Springer-Verlag, 2005.
[18] T. H. Cormen, Introduction to Algorithms. Cambridge, MA: MIT Press, 2001.

[19] Z. Han, A. L. Swindlehurst, and K. J. R. Liu, "Smart deployment/ movement of unmanned air vehicle to improve connectivity in MANET," in Proc. IEEE Wireless Commun. Netw. Conf., 2006, pp. 252-257.

[20] J. E. Wieselthier, G. D. Nguyen, and A. Ephremides, "On the construction of energy-efficient broadcast and multicast trees in wireless networks," in Proc. IEEE INFOCOM, Tel-Aviv, Israel, Mar. 2000, vol. 2, pp. 585-594.

[21] L. A. Santalo, Integral Geometry and Geometric Probability. Reading, MA: Addison-Wesley, 1976

[22] S. Boyd and L. Vandenberghe, Convex Optimization, Cambridge, U.K.: Cambridge Univ. Press, 2006. [Online]. Available: http://www. stanford.edu/ boyd/cvxbook.html

[23] G. W. Flake, R. E. Tarjan, and K. Tsioutsiouliklis, "Minimum cut tree clustering," in Proc. 1st WAW, Vancouver, BC, Canada, 2002.

[24] G. Golub, Matrix Computations, 3rd ed. Baltimore, MD: Johns Hopkins Univ. Press, 1996.

[25] B. Krishnamachari, Networking Wireless Sensors. Cambridge, U.K.: Cambridge Univ. Press, 2005.

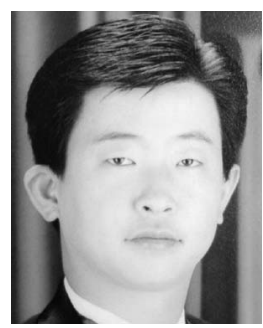

Zhu Han (S'01-M'04) received the B.S. degree in electronic engineering from Tsinghua University, Beijing, China, in 1997 and the M.S. and Ph.D. degrees in electrical engineering from the University of Maryland, College Park, in 1999 and 2003, respectively.

From 2000 to 2002, he was an R\&D Engineer with JDSU, Germantown, MD. From 2002 to 2003 , he was a Graduate Research Assistant with the University of Maryland. From 2003 to 2006, he was a Research Associate with the University of Maryland. From 2006 to 2008, he was an assistant professor with Boise State University, Boise, ID. Currently, he is an Assistant Professor with the Electrical and Computer Engineering Department, the University of Houston, Houston, TX. From June to August 2006, he was a visiting scholar with Princeton University, Princeton, NJ. From May to August 2007, he was a visiting professor with Stanford University, Stanford, CA. From May to August 2008, he was a visiting professor with the University of Oslo, Oslo, Norway and Supelec, Paris, France. His research interests include wireless resource allocation and management, wireless communications and networking, game theory, wireless multimedia, and security.

Dr. Han was the MAC Symposium vice chair of the 2008 IEEE Wireless Communications and Networking Conference. He was the Guest Editor for the Special Issue on Fairness of Radio Resource Management Techniques in Wireless Networks of the EURASIP Journal on Wireless Communications and Networking and the Special Issue on Game Theory of the EURASIP Journal on Advances in Signal Processing. He is a member of the Technical Programming Committee for the IEEE International Conference on Communications, the IEEE Vehicular Technology Conference, the IEEE Consumer Communications and Networking Conference, the IEEE Wireless Communications and Networking Conference, and the IEEE Globe Communication Conference. 


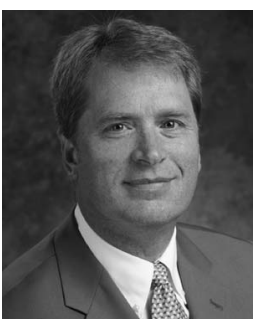

A. Lee Swindlehurst (S'83-M'84-SM'99-F'04) received the B.S. (summa cum laude) and M.S. degrees in electrical engineering from Brigham Young University, Provo, Utah, in 1985 and 1986, respectively, and the Ph.D. degree in electrical engineering from Stanford University, Stanford, CA, in 1991.

From 1986-1990, he was with ESL, Inc., Sunnyvale, CA, where he was involved in the design of algorithms and architectures for several radar and sonar signal processing systems. He was on the faculty of the Department of Electrical and Computer Engineering at Brigham Young University from 1990 to 2007, where he was a Full Professor and served as Department Chair from 2003 to 2006. During 1996-1997, he held a joint appointment as a visiting scholar at both Uppsala University, Uppsala, Sweden, and at the Royal Institute of Technology, Stockholm, Sweden. From 2006 to 2007, he was on leave working as Vice President of Research for ArrayComm LLC, San Jose, CA. He is currently a Professor of electrical engineering and computer science with the University of California at Irvine. His research interests include sensor array signal processing for radar and wireless communications, detection and estimation theory, and system identification, and he has over 170 publications in these areas.

Dr. Swindlehurst is a past Secretary of the IEEE Signal Processing Society. $\mathrm{He}$ is currently serving as Editor-in-Chief of the IEEE JOURNAL ON SELECTED TOPICS IN Signal Processing, as a member of the Editorial Boards for the EURASIP Journal on Wireless Communications and Networking and the IEEE Signal Processing Magazine, and is a past Associate Editor for the IEEE TRANSACTIONS ON SIgNAL PROCESSING. He is a recipient of several paper awards: the 2000 IEEE W. R. G. Baker Prize Paper Award, the 2006 IEEE Signal Processing Society's Best Paper Award, the 2006 IEEE Communications Society Stephen O. Rice Prize in the Field of Communication Theory, and he is co-author of a paper that received the IEEE Signal Processing Society Young Author Best Paper Award in 2001.

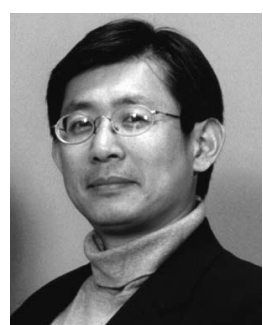

K. J. Ray Liu (F'03) is a Distinguished ScholarTeacher with the University of Maryland, College Park. He is Associate Chair of Graduate Studies and Research with the Electrical and Computer Engineering Department and leads the Maryland Signals and Information Group, conducting research encompassing broad aspects of information technology, including communications and networking, information forensics and security, multimedia signal processing, and biomedical technology. His recent books include Cooperative Communications and Networking (Cambridge University Press, 2008); Resource Allocation for Wireless Networks: Basics, Techniques, and Applications (Cambridge University Press, 2008), Ultra-Wideband Communication Systems: The Multiband OFDM Approach (IEEE-Wiley, 2007), Network-Aware Security for Group Communications (Springer, 2007), Multimedia Fingerprinting Forensics for Traitor Tracing (Hindawi, 2005), and Handbook on Array Processing and Sensor Networks (IEEE-Wiley, 2009).

Dr. Liu has received numerous honors and awards, including best paper awards from IEEE Signal Processing Society (twice), IEEE Vehicular Technology Society, and EURASIP; IEEE Signal Processing Society Distinguished Lecturer, the EURASIP Meritorious Service Award, and the National Science Foundation Young Investigator Award. He has also received various teaching and research recognitions from the University of Maryland, including university-level Invention of the Year Award and the Poole and Kent Senior Faculty Teaching Award and Outstanding Faculty Research Award, both from A. James Clark School of Engineering Faculty. Dr. Liu is a Fellow of the AAAS. He is Vice President-Publications and on the Board of Governor of the IEEE Signal Processing Society. He was the Editor-in-Chief of IEEE Signal Processing Magazine and the founding Editor-in-Chief of EURASIP Journal on Applied Signal Processing. 